\title{
Uma Solução Exata Mais Eficiente para a Soma de Variáveis Aleatórias Nakagami-m e Aplicações
}

\author{
Fernando Darío Almeida García, Francisco Raimundo Albuquerque Parente, Nathaly Orozco Garzón, \\ Henry Ramiro Carvajal Mora e José Cândido Silveira Santos Filho
}

\begin{abstract}
Resumo-Somas de variáveis aleatórias (VAs) desempenham um papel fundamental na análise de desempenho de sistemas de comunicação. Em particular, somas de VAs Nakagami- $m$ permitem caracterizar e avaliar o desempenho de receptores com combinação por ganho igual (EGC, do inglês equal-gain combining), uma técnica clássica de diversidade que tem sido explorada em redes móveis de quinta geração. Como a caracterização estatística de somas de variáveis - em termos da função densidade de probabilidade (PDF, do inglês probability density function) ou da função de distribuição acumulada (CDF, do inglês cumulative distribution function) - envolve um tratamento matemático bastante intrincado, as soluções exatas existentes para a soma de VAs Nakagami- $m$ são lentas, limitadas, ou mesmo inviáveis à medida que o número de VAs na soma aumenta. Neste trabalho, obtemos novas expressões exatas para a PDF e a CDF da soma de variáveis Nakagami- $m$, na forma de uma série infinita. Em comparação com o estado da arte, as novas expressões reduzem o custo computacional (ou, equivalentemente, o tempo de computação) em mais de $95 \%$. Para mostrar a aplicabilidade dessas expressões, analisamos a probabilidade de outage em receptores EGC. Simulações de Monte Carlo corroboram nossos resultados analíticos.
\end{abstract}

Palavras-Chave-Combinação por ganho igual, distribuição Nakagami- $m$, função densidade de probabilidade, função de distribuição acumulada, somas de variáveis aleatórias.

Abstract-Sums of random variables (RVs) play a key role in the performance analysis of communication systems. In particular, sums of Nakagami- $m$ RVs allow the performance characterization and evaluation of equal-gain combining (EGC) receivers, a classical diversity technique that has been explored in fifthgeneration mobile networks. As the statistical characterization of sums of variables - in terms of the probability density function (PDF) or the cumulative distribution function (CDF) - involves an intricate mathematical treatment, the exact solutions available for the sum of Nakagami- $m$ RVs are time-consuming, limited, or even unfeasible as the number of RVs in the sum increases. In this work, we obtain novel exact expressions for the PDF and CDF of the sum of Nakagami- $m$ variates, in terms of infinite series. Compared with the state of the art, the new expressions reduce the computational cost (or, equivalently, the computation time) by more than $95 \%$. To show the applicability of these expressions, we analyze the outage probability in EGC receivers. Monte-Carlo simulations corroborate our analytical results.

F. D. A. García, F. R. A. Parente e J. C. S. Santos Filho, Wireless Technology Laboratory, Departamento de Comunicações, Faculdade de Engenharia Elétrica e de Computação, Universidade Estadual de Campinas (UNICAMP), 13083-852, Campinas, SP, Brasil, e-mails \{ferdaral,parente,candido\}@ decom.fee.unicamp.br.

N. O. Garzón e H. R. C. Mora, Faculty of Engineering and Applied Sciences, Telecommunications Engineering, Universidad de Las Américas (UDLA), Quito, Ecuador, e-mails: \{nathaly.orozco,henry.carvajal\}@udla.edu.ec.

$\mathrm{O}$ trabalho de F. D. A. García foi financiado pela Fundação de Amparo à Pesquisa do Estado de São Paulo (FAPESP) com Processo No. 2021/03923-9. $\mathrm{O}$ trabalho de F. R. A. Parente foi financiado pela Fundação de Amparo à Pesquisa do Estado de São Paulo (FAPESP) com Processo No. 2018/25009-4.
Keywords - Cumulative distribution function, equal-gain combining, Nakagami- $m$ distribution, probability density function, sums of random variables.

\section{INTRODUÇÃO}

A análise de desempenho de vários sistemas de comunicação depende da caracterização estatística de somas de variáveis aleatórias (VAs). Por exemplo, somas de VAs aparecem em detecção de sinais, equalização, combinação de diversidade, e fenômenos de interferência [1]-[5]. Em particular, a soma de variáveis Nakagami- $m$ tem se mostrado adequada para modelar o desvanecimento do canal em sistemas de ondas milimétricas ${ }^{1}$ que utilizam combinação por ganho igual (EGC, do inglês equal-gain combining) [6]-[8], uma técnica clássica de diversidade amplamente explorada para melhorar o desempenho de redes convencionais e de quinta geração [9]-[11]. Nesses cenários, a análise de desempenho do sistema requer o conhecimento das estatísticas da soma, isto é, da função densidade de probabilidade (PDF, do inglês probability density function) ou, equivalentemente, da função de distribuição acumulada (CDF, do inglês cumulative distribution function). Todavia, o cálculo exato dessas funções para a soma de VAs Nakagami- $m$ é uma tarefa complicada, o que tem motivado a busca por soluções aproximadas.

Vários trabalhos propuseram soluções aproximadas para a soma de VAs Nakagami-m. De fato, foi o próprio Nakagami quem primeiro propôs aproximar a soma de variáveis Nakagami- $m$ independentes e identicamente distribuídas (i.i.d.) por outra variável Nakagami-m [12]. Com base nessa abordagem, uma distribuição aproximada para a soma de duas VAs Nakagami- $m$ correlacionadas e identicamente distribuídas foi proposta em [13]. As aproximações de [12] e [13] foram posteriormente estendidas em [14] para a soma de variáveis Nakagami- $m$ independentes, mas não necessariamente identicamente distribuídas (i.n.i.d.). Em [15], uma aproximação mais precisa para a PDF dessa soma foi obtida por meio de estimadores baseados em momentos da distribuição $\alpha-\mu$ [16]. Mais recentemente, com base em uma técnica denominada casamento de assíntotas, aproximações assintoticamente ótimas para a soma de VAs independentes e correlacionadas foram obtidas em [17] e [18], respectivamente.

Outros trabalhos recorreram a uma abordagem exata para fornecer soluções para a soma de VAs. Por exemplo, a PDF da soma exata de duas VAs Rayleigh independentes foi fornecida

${ }^{1}$ Ondas milimétricas referem-se a sinais de alta frequência usados na implementação de redes móveis de quinta geração $(5 \mathrm{G})$ [6]. 
em [19]. Em [20], uma representação em série infinita para essa mesma soma foi apresentada, mas, segundo os autores, problemas de convergência podem acontecer. Note que a PDF da soma de VAs Rayleigh é contemplada como caso particular neste trabalho, uma vez que a distribuição Rayleigh é uma condição especial da distribuição Nakagami- $m$. Com base nos resultados de [19], uma representação em termos de uma integral múltipla foi proposta em [21] para a soma de VAs Nakagami- $m$ i.n.i.d. No entanto, a solução em [21] requer um custo computacional muito elevado. Em [22], os autores apresentaram uma solução em forma fechada para a PDF da soma de duas VAs i.i.d. do tipo Nakagami- $m$. Essa solução foi estendida em [23] para um número arbitrário de VAs Nakagami- $m$ i.i.d., com a PDF da soma obtida na forma de séries infinitas aninhadas, cujo cálculo se torna difícil de tratar quando o número de variáveis excede, digamos, cinco ou seis. Uma solução em forma fechada para a PDF da soma de um número arbitrário de VAs Nakagami- $m$, mas dessa vez i.n.i.d., foi apresentada em [24] em termos da função hipergeométrica multivariada de Lauricella [25]. Infelizmente, essa função não se encontra implementada em pacotes computacionais de uso comercial. Além disso, uma vez que a definição padrão da função hipergeométrica multivariada de Lauricella depende de séries infinitas aninhadas [25, App. (A.20)], sua implementação está sujeita a um elevado custo computacional e a problemas de má convergência e instabilidade conforme o número de VAs na soma aumenta.

Neste trabalho, obtemos novas expressões exatas para a PDF e a CDF da soma de VAs Nakagami-m i.i.d., na forma de série infinita. Além disso, como exemplo de aplicação, analisamos o desempenho de receptores EGC, obtendo novas expressões exatas e assintóticas para a probabilidade de outage (OP, do inglês outage probability). Simulações de Monte Carlo corroboram a validade desses resultados. Nossas expressões fornecem uma solução mais tratável e computacionalmente mais barata que o estado da arte [24], reduzindo o tempo de cálculo em mais de $95 \%$. Além disso, ao contrário de soluções anteriores, a complexidade matemática das expressões propostas não aumenta com o número de VAs na soma.

$\mathrm{O}$ restante do artigo está organizado como segue. A Seção II apresenta a formulação do problema. A Seção III apresenta a PDF e a CDF da soma de VAs i.i.d. Nakagami-m. A Seção IV analisa o desempenho de receptores EGC. A Seção V discute resultados numéricos. Finalmente, a Seção VI apresenta as principais conclusões do trabalho.

\section{FORMULAÇÃO DO PROBlEMA}

Seja $Z$ a soma de $N$ VAs $X_{n}$ i.i.d. do tipo Nakagami- $m$ :

$$
Z=\sum_{n=1}^{N} X_{n} .
$$

Cada somante $X_{n}$ tem $\mathrm{PDF} f_{X_{n}}(\cdot)$ e $\mathrm{CDF} F_{X_{n}}(\cdot)$ dadas, respectivamente, por

$$
\begin{aligned}
f_{X_{n}}\left(x_{n}\right) & =\frac{2 x_{n}^{2 m-1} m^{m}}{\Omega^{m} \Gamma(m)} \exp \left(-\frac{m x_{n}^{2}}{\Omega}\right) \\
F_{X_{n}}\left(x_{n}\right) & =\frac{\Gamma\left(m, \frac{m x_{n}^{2}}{\Omega}\right)}{\Gamma(m)}
\end{aligned}
$$

em que $m \geq 1 / 2$ é um parâmetro de forma, $x_{n}>0$, $\Omega \triangleq \mathbb{E}\left[X_{n}^{2}\right] \forall n, \mathbb{E}[\cdot]$ denota valor esperado, $\Gamma(\cdot)$ é a função gama [26, eq. (5.2.1)], e $\Gamma(\cdot, \cdot)$ é função gama incompleta superior [26, eq. (8.2.2)].

Nosso principal objetivo é obter a PDF e a CDF da soma em (1) de forma exata, tratável, e computacionalmente barata. Esse objetivo é cumprido na próxima seção.

\section{EstatísticAS DA SOMA}

Nesta seção, deduzimos novas representações em termos de séries para a PDF e a CDF da soma de VAs i.i.d. do tipo Nakagami- $m$. As representações obtidas são mais simples e convergem mais rapidamente que as soluções disponíveis na literatura. A abordagem está baseada no cálculo de resíduos e na transformada de Laplace, como detalhado a seguir.

\section{A. A PDF da Soma}

Com o intuito de obter a PDF da soma $Z$ em (1), inicialmente aplicamos a transformada de Laplace à PDF marginal de cada $X_{n}$, ou seja,

$$
\mathcal{L}\left\{f_{X_{n}}\right\}(s)=\int_{0}^{\infty} \exp \left(-s x_{n}\right) f_{X_{n}}\left(x_{n}\right) \mathrm{d} x_{n},
$$

em que $s \in \mathbb{C}$.

Substituindo-se (2) em (4), e depois de manipulações algébricas, (4) reduz-se a

$$
\begin{aligned}
\mathcal{L}\left\{f_{X_{n}}\right\}(s)= & \frac{2 m^{m}}{\Omega^{m} \Gamma(m)} \int_{0}^{\infty} x_{n}^{2 m-1} \exp \left(-s x_{n}\right) \\
& \times G_{1,0}^{0,1}\left[\begin{array}{c|c}
- & m x_{n}^{2} \\
\Omega
\end{array}\right] \mathrm{d} x_{n},
\end{aligned}
$$

em que $G_{u, v}^{p, q}[\cdot]$ denota a função $G$ de Meijer [26, eq. (16.17.1)].

Aplicando [27, eq. (07.34.02.0001.01)] junto com simplificações matemáticas, podemos expressar (5) como

$$
\begin{aligned}
\mathcal{L}\left\{f_{X_{n}}\right\}(s)= & \frac{2 m^{m}}{\Omega^{m} \Gamma(m)}\left(\frac{1}{2 \pi j}\right) \int_{0}^{\infty} \exp \left(-s x_{n}\right) \\
& \times \oint_{\mathcal{L}_{\mathrm{s}, 1}} \Gamma\left(s_{1}\right) x_{n}^{2 m-1}\left(\frac{m x_{n}^{2}}{\Omega}\right)^{-s_{1}} \mathrm{~d} s_{1} \mathrm{~d} x_{n},
\end{aligned}
$$

em que $j=\sqrt{-1}, s_{1}$ é uma variável complexa de integração, e $\mathcal{L}_{\mathbf{s}, 1}$ é um contorno de integração que começa no ponto $-\infty+j \psi_{1}$ e termina no ponto $-\infty+j \psi_{2}\left(-\infty<\psi_{2}<\right.$ $\left.\psi_{1}<+\infty\right)$ de forma que $\mathcal{L}_{\mathbf{s}, 1}$ circunde todos os pólos de $\Gamma\left(s_{1}\right)[25]$.

Como $\int_{0}^{\infty}\left|\exp \left(-s x_{n}\right) f_{X_{n}}\left(x_{n}\right)\right| \mathrm{d} x_{n}<\infty$, podemos invocar o teorema de Fubini [28] para inverter a ordem de integração em (6). Dessa forma, depois de se avaliar a integral interna, obtém-se

$$
\begin{aligned}
\mathcal{L}\left\{f_{X_{n}}\right\}(s)= & \frac{2 m^{m} s^{-2 m}}{\Omega^{m} \Gamma(m)}\left(\frac{1}{2 \pi j}\right) \oint_{\mathcal{L}_{\mathbf{s}, 1}^{\dagger}} \Gamma\left(2 m-2 s_{1}\right) \\
& \times \Gamma\left(s_{1}\right)\left(\frac{m}{s^{2} \Omega}\right)^{-s_{1}} \mathrm{~d} s_{1},
\end{aligned}
$$

em que $\mathcal{L}_{\mathrm{s}, 1}^{\dagger}$ é um novo contorno de integração, uma vez que a integração em $x_{n}$ mudou o núcleo da integração em $s_{1}$. Especificamente, $\mathcal{L}_{\mathbf{s}, 1}^{\dagger}$ é um contorno que começa no ponto $\xi-$ $j \infty$ e vai até $\xi+j \infty(\xi \in(0, \infty))$ de forma que todos os pólos de $\Gamma\left(s_{1}\right)$ estejam separados daqueles de $\Gamma\left(2 m-2 s_{1}\right)$ [25]. 
Utilizando o teorema do resíduo de Cauchy, podemos expressar (7) como segue [29]:

$$
\mathcal{L}\left\{f_{X_{n}}\right\}(s)=\frac{2 m^{m} s^{-2 m}}{\Omega^{m} \Gamma(m)} \sum_{i=0}^{\infty} \operatorname{Res}\left[\Xi_{1}\left(s_{1}\right) ;\left\{s_{1}=-i\right\}\right],
$$

em que Res $\left[\Xi_{1}\left(s_{1}\right) ;\left\{s_{1}=-i\right\}\right]$ denota o resíduo da função $\Xi_{1}\left(s_{1}\right)=\Gamma\left(2 m-2 s_{1}\right) \Gamma\left(s_{1}\right)\left(\frac{m}{s^{2} \Omega}\right)^{-s_{1}}$ avaliada nos pólos $s_{1}=-i$.

Depois de aplicar a operação de resíduo [29, eq. (16.3.3)], obtemos

$$
\mathcal{L}\left\{f_{X_{n}}\right\}(s)=\frac{2 m^{m} s^{-2 m}}{\Omega^{m} \Gamma(m)} \sum_{i=0}^{\infty} \frac{\Gamma(2(i+m))\left(-\frac{m}{s^{2} \Omega}\right)^{i}}{i !} .
$$

Para o caso de VAs independentes, a PDF da soma é dada pela convolução das PDFs marginais dos somantes, isto é,

$$
f_{Z}(z)=f_{X_{1}}\left(x_{1}\right) * f_{X_{2}}\left(x_{2}\right) * \cdots * f_{X_{N}}\left(x_{N}\right) .
$$

Podemos então aplicar a transformada de Laplace em ambos os lados de (10) e usar o fato de que as VAs $\left\{X_{n}\right\}_{n=1}^{N}$ são identicamente distribuídas. Isso conduz a

$$
\mathcal{L}\left\{f_{Z}\right\}(s)=\left(\mathcal{L}\left\{f_{X_{n}}\right\}(s)\right)^{N} .
$$

Para obter a PDF soma em (1), precisamos agora calcular a transformada de Laplace inversa de (11), definida em termos da integral de Fourier-Mellin como [30, eq. (4.3)]

$$
f_{Z}(z)=\left(\frac{1}{2 \pi j}\right) \oint_{\mathcal{L}_{\mathbf{s}, 2}} \exp (s z) \mathcal{L}\left\{f_{Z}\right\}(s) \mathrm{d} s,
$$

em que $\mathcal{L}_{\mathrm{s}, 2}$ é o contorno de Bromwich.

Substituindo (9) em (11), e a expressão resultante em (12), obtemos

$$
\begin{aligned}
f_{Z}(z)= & \left(\frac{2 m^{m}}{\Omega^{m} \Gamma(m)}\right)^{N}\left(\frac{1}{2 \pi j}\right) \oint_{\mathcal{L}_{\mathrm{s}, 2}} s^{-2 N m} \exp (s z) \\
& \times(\sum_{i=0}^{\infty} s^{-2 i} \overbrace{\frac{\left(-\frac{m}{\Omega}\right)^{i} \Gamma(2(i+m))}{i !}}^{a_{i}})^{N} \mathrm{~d} s .
\end{aligned}
$$

Em (13), para facilitar a inversão de Laplace, interessa obter uma equivalência do tipo

$$
\left(\sum_{i=0}^{\infty} s^{-2 i} a_{i}\right)^{N}=\sum_{i=0}^{\infty} s^{-2 i} c_{i}
$$

Ao derivarmos (14) com relação a $s^{-2}$, notamos que os coeficientes $c_{i}$ podem ser obtidos recursivamente por

$$
\begin{aligned}
c_{0} & =\Gamma(2 m)^{N} \\
c_{i} & =\frac{1}{i \Gamma(2 m)} \sum_{l=1}^{i} \frac{c_{i-l}(l N-i+l)\left(-\frac{m}{\Omega}\right)^{l} \Gamma(2(l+m))}{l !} .
\end{aligned}
$$

Finalmente, substituindo (14) e (15) em (13), e após uma inversão termo a termo com a ajuda de [30, Appendix A], obtemos uma representação em série para a PDF da soma:

$$
f_{Z}(z)=\left(\frac{2 m^{m}}{\Omega^{m} \Gamma(m)}\right)^{N} \sum_{i=0}^{\infty} \frac{c_{i} z^{2(i+N m)-1}}{\Gamma(2(i+N m))} .
$$

Pode-se facilmente provar que devido ao fator $\Gamma(2(i+N m))$, (16) converge absolutamente. Assim, (16) converge $\forall(m, \Omega)$.

\section{B. A CDF da Soma}

A CDF da soma $Z$ pode ser obtida de (16) via definição:

$$
\begin{aligned}
F_{Z}(z) & \triangleq \int_{0}^{z} f_{Z}(u) \mathrm{d} u \\
& =\left(\frac{2 m^{m}}{\Omega^{m} \Gamma(m)}\right)^{N} \int_{0}^{z} \sum_{i=0}^{\infty} \frac{c_{i} u^{2(i+N m)-1}}{\Gamma(2(i+N m))} \mathrm{d} u .
\end{aligned}
$$

Intercambiando a ordem da integração e do somatório, e avaliando a integral resultante, finalmente obtemos

$$
F_{Z}(z)=\left(\frac{2 m^{m}}{\Omega^{m} \Gamma(m)}\right)^{N} \sum_{i=0}^{\infty} \frac{c_{i} z^{2(i+N m)}}{\Gamma(2(i+N m)+1)} .
$$

O intercâmbio na ordem das operações é justificado pela convergência absoluta de (18). Pode-se provar que devido ao fator $\Gamma(2(i+N m)+1)$, (18) converge absolutamente.

As expressões (16) e (18) são as principais contribuições analíticas deste trabalho.

\section{APLICAÇÃO AO EGC}

Em um sistema EGC pré-detecção de $N$ ramos de diversidade e com níveis de ruído iguais em cada ramo, a relação sinal-ruído (SNR) instantânea é dada por [31]

$$
\Phi=\frac{E_{s}}{N N_{0}}\left(\sum_{n=1}^{N} X_{n}\right)^{2}=\frac{E_{s}}{N N_{0}} Z^{2},
$$

em que $\left\{X_{n}\right\}_{n=1}^{N}$ são as envoltórias dos ramos (VAs Nakagami- $m$ i.i.d., por hipótese), $E_{s}$ é a energia média recebida por símbolo, $N_{0}$ é a densidade espectral da potência de ruído unilateral, e $E_{s} / N_{0}$ é a SNR média por símbolo.

Antes de prosseguir com a análise de OP para o EGC, é conveniente deduzir a PDF e a CDF da SNR instantânea $(\Phi)$. A partir de (16), a PDF de $\Phi$ pode ser obtida por meio de uma transformação simples de variáveis, como

$$
f_{\Phi}(\phi)=\frac{N N_{0}}{2 E_{s}}\left(\frac{2 m^{m}}{\Gamma(m) \Omega^{m}}\right)^{N} \sum_{i=0}^{\infty} \frac{c_{i}\left(\frac{\phi N N_{0}}{E_{s}}\right)^{i+m N-1}}{\Gamma(2 i+2 N m)} .
$$

Além disso, de (20), a CDF de $\Phi$ pode ser obtida como

$$
\begin{aligned}
F_{\Phi}(\phi) & \triangleq \int_{0}^{\phi} f_{\Phi}(u) \mathrm{d} u \\
& =\left(\frac{2 m^{m}}{\Gamma(m) \Omega^{m}}\right)^{N} \sum_{i=0}^{\infty} \frac{c_{i}\left(\frac{\phi N N_{0}}{E_{s}}\right)^{i+m N}}{\Gamma(2 i+2 N m+1)} .
\end{aligned}
$$

A OP de um sistema de comunicação é definida como a probabilidade de a SNR instantânea se encontrar abaixo de um certo limiar (digamos, $\gamma_{\text {th }}$ ). Portanto, para o EGC, a OP pode ser obtida diretamente de (21):

$$
P_{\text {out }} \triangleq \operatorname{Pr}\left[\Phi \leq \gamma_{\mathrm{th}}\right]=\left(\frac{2 m^{m}}{\Gamma(m) \Omega^{m}}\right)^{N} \sum_{i=0}^{\infty} \frac{c_{i}\left(\frac{\gamma_{\mathrm{th}} N N_{0}}{E_{s}}\right)^{i+m N}}{\Gamma(2 i+2 N m+1)},
$$

em que $\operatorname{Pr}[\cdot]$ denota probabilidade. Em particular, uma condição de grande interesse prático é a de alta SNR média, na qual os sistemas operam de forma mais confiável. Nessa região, como de praxe, podemos considerar $E_{s} / N_{0} \rightarrow \infty$, de modo que a OP assintótica correspondente pode ser obtida a partir do primeiro termo $(i=0)$ na soma em (22) [32], ou seja,

$$
P_{\mathrm{out}} \sim\left\{\frac{\Omega}{m N \gamma_{\mathrm{th}}}\left[\frac{\Gamma(m)^{N} \Gamma(2 m N+1)}{c_{0} 2^{N}}\right]^{\frac{1}{m N}} \frac{E_{s}}{N_{0}}\right\}^{-m N},
$$




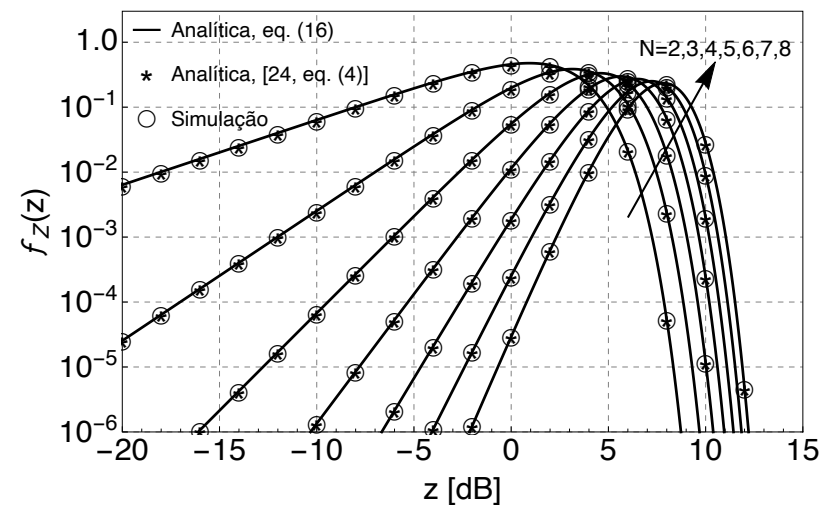

Fig. 1. PDF de $Z$ para $m=0.5, \Omega=1$ e diferentes valores de $N$.

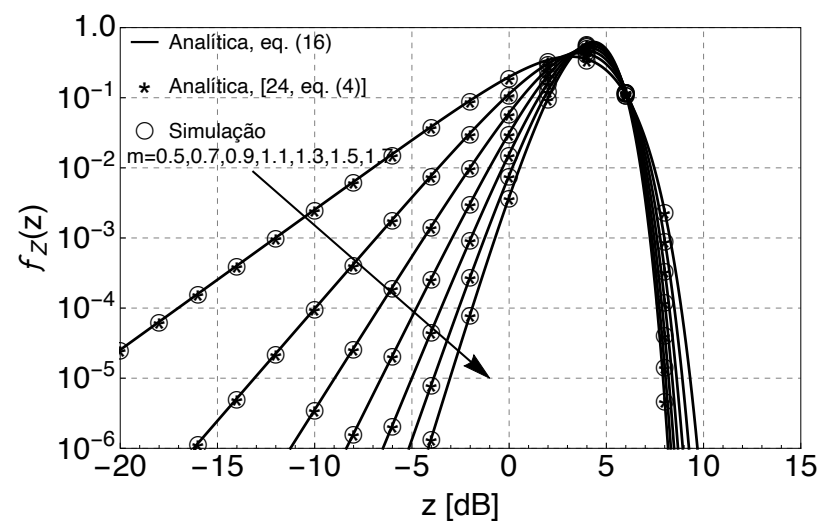

Fig. 2. PDF de $Z$ para $\Omega=1, N=3$ e diferentes valores de $m$.

em que o símbolo $\sim$ denota equivalência assintótica (para $\left.E_{s} / N_{0} \rightarrow \infty\right)$. Portanto, o ganho de codificação $O_{\mathrm{c}}$ e o ganho de diversidade $O_{\mathrm{d}}$ de $P_{\text {out }} \sim\left(O_{\mathrm{c}} E_{s} / N_{0}\right)^{-O_{\mathrm{d}}}$ são dados por

$$
\begin{aligned}
& O_{\mathrm{c}}=\frac{\Omega}{m N \gamma_{\mathrm{th}}}\left[\frac{\Gamma(m)^{N} \Gamma(2 m N+1)}{c_{0} 2^{N}}\right]^{\frac{1}{m N}} \\
& O_{\mathrm{d}}=m N .
\end{aligned}
$$

As expressões (20)-(24) são contribuições originais.

\section{Resultados Numéricos E Discussões}

Nesta seção, validamos nossas expressões via simulações de Monte Carlo. O número de realizações foi fixado em $10^{7}$. Além disso, ilustramos a eficiência de (16) em comparação com o estado da arte [24, eq. (4)], considerando a economia de tempo obtida pela nossa proposta. Vale lembrar que a PDF em [24, eq. (4)] é dada em termos da função hipergeométrica multivariada de Lauricella, indisponível de forma nativa em pacotes de computação comerciais, tendo portanto que ser implementada via séries infinitas aninhadas [25, App. (A.20)].

As Figs. 1 e 2 mostram as PDFs $f_{Z}(\cdot)$ analítica e simulada. $\mathrm{Na}$ Fig. 1 , consideramos $m=0.5, \Omega=1$, e diferentes valores de $N$, enquanto que na Fig. 2 consideramos $\Omega=1, N=3$, e diferentes valores de $m$. Um tanto quanto arbitrariamente, usamos 115 termos em (16). Em ambas as figuras, observe a concordância perfeita entre a solução exata em (16) e os resultados de simulação.

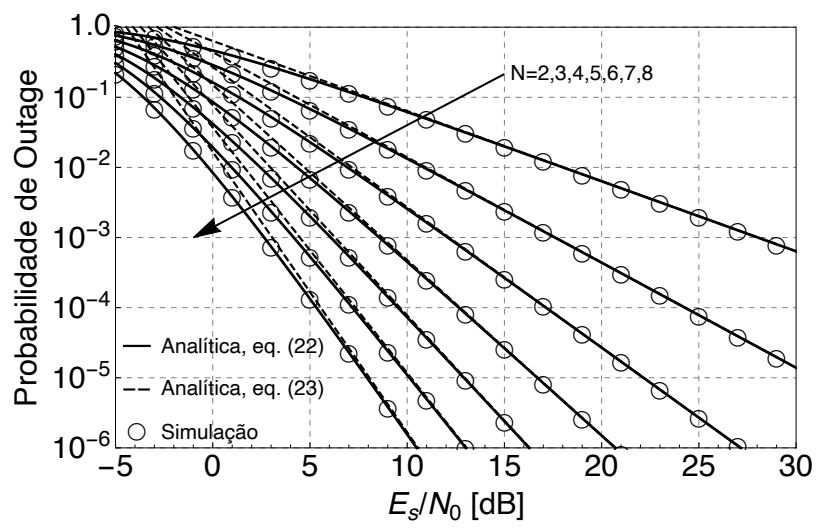

Fig. 3. OP em receptores EGC para $m=0.5, \Omega=1, \gamma_{\text {th }}=0 \mathrm{~dB}$ e diferentes valores de $N$.

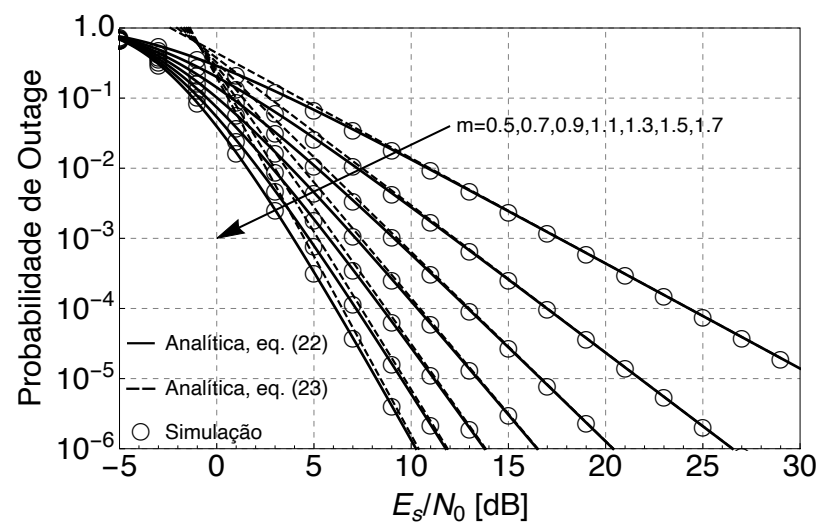

Fig. 4. OP em receptores EGC para $N=3, \Omega=1, \gamma_{\text {th }}=0 \mathrm{~dB}$ e diferentes valores de $m$.

As Figs. 3 e 4 mostram a OP para receptores EGC em função da SNR média por símbolo. Na Fig. 3, consideramos $m=0.5, \Omega=1, \gamma_{\mathrm{th}}=0 \mathrm{~dB}$, e diferentes valores de $N$, enquanto que na Fig. 4 consideramos $N=3, \Omega=1, \gamma_{\text {th }}=0$ $\mathrm{dB}$, e diferentes valores de $m$. Novamente, veja como a solução exata em (22) está em pleno acordo com as simulações. Além disso, veja como a solução assintótica em (23), além de muito simples, fornece uma excelente aproximação para valores médios a altos de SNR.

Para ilustrar a eficiência de (16), selecionamos diferentes cenários, mostrados na Tabela I. Cada linha apresenta (i) o valor de referência de $f_{Z}(z)$, calculado via convolução numérica na plataforma Mathematica; (ii) o número mínimo de termos em (16) para garantir um erro relativo menor que $10^{-5}$; (iii) o tempo de computação para (16) com esse número de termos; (iv) o tempo de computação para [24, eq. (4)] com termos suficientes para garantir um erro relativo também menor que $10^{-5}$; e (v) a economia de tempo de (16) em relação a [24, eq. (4)]. Note que nenhum cenário exigiu mais que 25 termos em (16). Além disso, note que o tempo de computação de [24, eq. (4)] cresce à medida que o número $N$ de somantes aumenta, enquanto que o tempo de computação de (16) pouco varia com $N$. Por fim, e não menos importante, note que, em relação a [24, eq. (4)], (16) traz uma economia de tempo de computação superior a $95 \%$. 
TABELA I

EFICIÊNCIA DE (16) COMPARADA À DE [24, EQ. (4)] (ERRO RELATIVO $<10^{-5}$ )

\begin{tabular}{|c|c|c|c|c|c|}
\hline Configuração de parâmetros & $f_{Z}(z)$ & $\begin{array}{c}\text { Número de termos } \\
\text { para (16) }\end{array}$ & $\begin{array}{c}\text { Tempo de computação } \\
\text { para (16) }[s]\end{array}$ & $\begin{array}{l}\text { Tempo de computação } \\
\text { para }[24, \text { eq. }(4)][s]\end{array}$ & $\begin{array}{c}\text { Economia } \\
\text { de tempo [\%] }\end{array}$ \\
\hline$m=0.5, \Omega=1, N=4, z=3$ & 0.3315 & 17 & 0.0014 & 0.0273 & 95.2016 \\
\hline$m=0.5, \Omega=1, N=5, z=3$ & 0.2604 & 17 & 0.0013 & 0.1594 & 99.1764 \\
\hline$m=1.0, \Omega=1, N=5, z=3$ & 0.1620 & 19 & 0.0018 & 0.8471 & 99.7855 \\
\hline$m=1.5, \Omega=1, N=5, z=3$ & 0.0804 & 25 & 0.0035 & 2.7922 & 99.8738 \\
\hline$m=0.5, \Omega=1, N=6, z=3$ & 0.1516 & 16 & 0.0011 & 1.1596 & 99.9011 \\
\hline$m=1.0, \Omega=1, N=6, z=3$ & 0.0376 & 19 & 0.0015 & 13.425 & 99.9883 \\
\hline$m=1.5, \Omega=1, N=6, z=3$ & 0.0073 & 20 & 0.0027 & 39.744 & 99.9932 \\
\hline$m=0.5, \Omega=1, N=7, z=3$ & 0.0701 & 16 & 0.0011 & 9.8613 & 99.9883 \\
\hline$m=1.0, \Omega=1, N=7, z=3$ & 0.0056 & 16 & 0.0013 & 165.241 & 99.9992 \\
\hline$m=1.5, \Omega=1, N=7, z=3$ & 0.0003 & 16 & 0.0081 & 158.015 & 99.9948 \\
\hline
\end{tabular}

\section{CONCLUSÕES}

Neste artigo, obtivemos novas expressões exatas para a PDF e a CDF da soma de VAs Nakagami- $m$ i.i.d. Além de compactas, as novas expressões exigem um esforço computacional significativamente menor que o das soluções disponíveis. Como aplicação, obtivemos novas expressões exatas e assintóticas, também compactas e eficientes, para a OP de receptores EGC operando em ambiente Nakagami- $m$.

\section{REFERÊNCIAS}

[1] N. C. Beaulieu and A. A. Abu-Dayya, "Analysis of equal gain diversity on Nakagami fading channels," IEEE Trans. Commun., vol. 39, no. 2, pp. 225-234, Feb. 1991.

[2] A. Annamalai, C. Tellambura, and V. K. Bhargava, "Equal-gain diversity receiver performance in wireless channels," IEEE Trans. Commun., vol. 48 , no. 10 , pp. 1732-1745, Oct. 2000.

[3] M.-S. Alouini and M. K. Simon, "Performance analysis of coherent equal gain combining over Nakagami- $m$ fading channels," IEEE Trans. Veh. Technol., vol. 50, no. 6, pp. 1449-1463, Nov. 2001.

[4] H. C. Mora, N. V. O. Garzón, F. D. A. García, and C. de Almeida "On the bit error rate of OFDMA employing short cyclic prefix and maximal ratio combining," in Proc. IEEE Colombian Conference on Communications and Computing (COLCOM), Aug. 2019, pp. 1-6.

[5] F. D. A. García, A. C. F. Rodriguez, G. Fraidenraich, and J. C. S. Santos Filho, "CA-CFAR detection performance in homogeneous Weibull clutter," IEEE Geosci. Remote Sens. Lett., vol. 16, no. 6, pp. 887-891, Jun. 2019.

[6] T. R. R. Marins, A. A. dos Anjos, V. M. R. Peñarrocha, L. Rubio, J. Reig, R. A. A. de Souza, and M. D. Yacoub, "Fading evaluation in the mmwave band," IEEE Trans. on Comm., vol. 67, no. 12, pp. 8725-8738, Dec. 2019.

[7] S. M. Gulfam, S. J. Nawaz, K. B. Baltzis, A. Ahmed, and N. M. Khan, "Characterization of fading statistics of mmwave $(28 \mathrm{GHz}$ and $38 \mathrm{GHz}$ ) outdoor and indoor radio propagation channels," MDPI Technologies, vol. 7, no. 1, pp. 1-16, Jan. 2019.

[8] G. Yang, M. Xiaoa, and Z. Pang, "Delay analysis of traffic dispersion with nakagami-m fading in millimeter-wave bands," in IEEE Wireless Comm. and Net. Conf. (WCNC), Barcelona, Spain, Oct. 2018, pp. 1-6.

[9] H. Hashemi, J. Haghighat, M. Eslami, and W. A. Hamouda, "Analysis of equal gain combining over fluctuating two-ray channels with applications to millimeter-wave communications," IEEE Trans. Veh. Technol., vol. 69, no. 2, pp. 1751-1765, Feb. 2020.

[10] S. Schwarz, E. Zöchmann, M. Müller, and K. Guan, "Dependability of directional millimeter wave vehicle-to-infrastructure communications," IEEE Access, vol. 8, pp. 53 162-53 171, Mar. 2020.

[11] G. R. MacCartney and T. S. Rappaport, "Millimeter-wave base station diversity for 5G coordinated multipoint (CoMP) applications," IEEE Trans. Wireless Commun., vol. 18, no. 7, pp. 3395-3410, Jul. 2019.

[12] M. Nakagami, "The $m$-distribution-A general formula of intensity distribution of rapid fading," Stat. Methods Radio Wave Propag., vol. 26, no. 2, p. 3-36, Apr. 1960.

[13] J. Reig and N. Cardona, "Nakagami- $m$ approximate distribution of sum of two Nakagami- $m$ correlated variables," Electron. Lett., vol. 36, no. 11, pp. 978-980, May 2000.
[14] J. C. S. Santos Filho and M. D. Yacoub, "Nakagami- $m$ approximation to the sum of $M$ non-identical independent Nakagami- $m$ variates," Electron. Lett., vol. 40, no. 15, pp. 951-952, Jul. 2004.

[15] D. B. da Costa, M. D. Yacoub, and J. C. S. Santos Filho, "An improved closed-form approximation to the sum of arbitrary Nakagami$m$ variates," IEEE Trans. Veh. Technol., vol. 57, no. 6, pp. 3854-3858, Nov. 2008.

[16] M. D. Yacoub, "The $\alpha-\mu$ distribution: A physical fading model for the stacy distribution," IEEE Trans. Veh. Technol., vol. 56, no. 1, pp. 27-34, Jan. 2007.

[17] V. Perim, J. D. V. Sánchez, and J. C. S. Santos Filho, "Asymptotically exact approximations to generalized fading sum statistics," IEEE Trans. Wireless Commun., vol. 19, no. 1, pp. 205-217, Jan. 2020.

[18] F. R. A. Parente and J. C. S. Santos Filho, "Asymptotically exact framework to approximate sums of positive correlated random variables and application to diversity-combining receivers," Wireless Commun. Lett., vol. 8, no. 4, pp. 1012-1015, Aug. 2019

[19] D. G. Brennan, "Linear diversity combining techniques," Proc. IRE, vol. 47, pp. 1075-1102, Jun. 1959.

[20] N. C. Beaulieu, "An infinite series for the computation of the complementary probability distribution function of a sum of independent random variables and its application to the sum of Rayleigh random variables," IEEE Trans. Commun., vol. 38, no. 9, pp. 1463-1474, Sep. 1990.

[21] M. D. Yacoub, C. R. C. M. da Silva, and J. E. Vargas Bautista, "Secondorder statistics for diversity-combining techniques in Nakagami-fading channels," IEEE Trans. Veh. Technol., vol. 50, no. 6, pp. 1464-1470, Nov. 2001

[22] C.-D. Iskander and P. T. Mathiopoulos, "Analytical level crossing rates and average fade durations for diversity techniques in Nakagami fading channels," IEEE Trans. Commun., vol. 50, no. 8, pp. 1301-1309, Aug. 2002.

[23] P. Dharmawansa, N. Rajatheva, and K. Ahmed, "On the distribution of the sum of Nakagami- $m$ random variables," IEEE Trans. Commun., vol. 55, no. 7, pp. 1407-1416, Jul. 2007.

[24] M. A. Rahman and H. Harada, "New exact closed-form PDF of the sum of Nakagami- $m$ random variables with applications," IEEE Trans. Commun., vol. 59, no. 2, pp. 395-401, Feb. 2011.

[25] A. M. Mathai, R. K. Saxena, and H. J. Haubold, The H-function: Theory and Applications. New York, NY: Springer, 2009.

[26] F. W. J. Olver, D. W. Lozier, R. F. Boisvert, and C. W. Clark, NIST Handbook of Mathematical Functions, 1st ed. Washington, DC: US Dept. of Commerce: National Institute of Standards and Technology (NIST), 2010

[27] Wolfram Research, Inc. (2018), Wolfram Research, Accessed: Sep. 19, 2020. [Online]. Available: http://functions.wolfram.com

[28] G. Fubini, "Sugli integrali multipli." Rom. Acc. L. Rend. (5), vol. 16, no. 1, pp. 608-614, 1907.

[29] E. Kreyszig, Advanced Engineering Mathematics, 10th ed. New Jersey, NJ: John Wiley \& Sons, 2010.

[30] J. L. Schiff, The Laplace Transform: Theory and Applications, 1st ed New York, NY: Springer, 1999.

[31] M. K. Simon and M.-S. Alouini, Digital Communication over Fading Channels, 2nd ed. Hoboken, NJ: Wiley, 2005.

[32] Z. Wang and G. B. Giannakis, "A simple and general parameterization quantifying performance in fading channels," IEEE Trans. Commun., vol. 51, no. 8, pp. 1389-1398, Aug. 2003. 